\title{
Study of the Magnetization Processes in Amorphous and Nanocrystalline FINEMET by the Numerical Decomposition of the Hysteresis Loops
}

\author{
J. KovÁC̆ ${ }^{a, *}$, L. NOVÁK ${ }^{b}$ AND L.K. VARGA ${ }^{c}$
}

${ }^{a}$ Institute of Experimental Physics, Slovak Academy of Sciences, Watsonova 47, 04001 Košice, Slovakia

${ }^{b}$ Department of Physics, Faculty of Electrical Engineering and Informatics, Technical University of Košice,

Letná 9, 04200 Košice, Slovakia

${ }^{c}$ Wigner Center for Physics, Institute for Solid State Physics and Optics, H-1525 Budapest, P.O. Box 49, Hungary

The magnetization processes in amorphous and nanocrystalline FINEMET ribbons were studied by the numerical decomposition of the quasi-static hysteresis loop to the contributions of the domain wall movement, the domain rotations, and the domain wall annihilation and nucleation processes following the hyperbolic $T(x)$ model of hysteresis. The hysteresis data measured during decrease of the excitation magnetic field were used for the separation of these processes. The significant differences in behavior of these two materials were found. In amorphous state the domain rotations component dominates whereas in nanocrystalline state the domain wall movement component prevails. These differences are reflected in the anisotropy field distributions as well.

DOI: 10.12693/APhysPolA.131.732

PACS/topics: 75.60.-d, 75.60.Ej

\section{Introduction}

The soft magnetic materials represent very important part of materials in research and industry over the last hundred years. The understanding of their magnetic behavior is necessary for further improvement of their magnetic properties in the newest applications. One way of investigation of these properties is modeling. There exist a lot of models which try to describe and simulate the basic magnetization processes in ferromagnetic media [1-4]. The models referring to soft and semihard magnetic materials assume that magnetization processes can be divided quite strictly to low-field (below coercive field) and high-field (above coercive field) regions. The domain wall movements (DWM) prevail in the low-field region whereas at high-field region the domain rotations (DR) dominate. However, for modern ultrasoft magnetic amorphous and nanocrystalline materials these two magnetization processes cannot be separated because of low effective crystalline anisotropy. Many of models try to describe the combined effect of DWM and DR processes within a common hysteresis loop [5]. Conversely, the two distinct physical processes can be separated in the whole excitation range, individually modeled and linearly superimposed [6]. Moreover, a third process can be added, the domain wall annihilation and nucleation (DWAN) comprising the high field region as well.

In this work, we try to compare the magnetization processes in amorphous and nanocrystalline states of Finemet type material. The hyperbolic $T(x)$ model $[3,6-10]$

*corresponding author; e-mail: jkovac@saske.sk will be used which enables the separation of the three processes (DWM, DR and DWAN) and subsequently their linear combination to model the hysteretic behavior of the material.

\section{Theoretical background}

The detailed description of $T(x)$ model is out of range of this work, it can be found elsewhere $[3,8]$. Here we restrict ourselves to the basic equations only. The experimental data obtained from descending branch of the hysteresis loop is fitted by

$$
\begin{aligned}
& m=\sum_{i=1}^{n}\left(A_{i} f_{ \pm i} \pm d_{i}\right), \\
& f_{ \pm i}=\tanh \left(\alpha_{i}\left(h \mp a_{0 i}\right)\right), \\
& d_{i}=\frac{A_{1}}{2}\left[f_{-i}\left(h_{m}\right)-f_{+i}\left(h_{m}\right)\right],
\end{aligned}
$$

where $m$ is the descending magnetization, $h$ - the applied magnetic field, $h_{m}$ - the maximum applied magnetic field, $A_{i}$ - the amplitude of the particular magnetization process, $\alpha_{i}$ - the sheering factor and $a_{0 i}$ - the coercivity of the $i$-th process $[6-10]$. These formulae can be used for the splitting of the measured hysteresis loop into two contributions [6] - DWM and DR - and into three contributions [7] adding a third contribution - DWAN.

\section{Experimental}

The amorphous samples were prepared by planar flow casting in Wigner Research Center for Physics, Budapest. The dimensions of samples were: $105 \mathrm{~mm}$ long, $5 \mathrm{~mm}$ wide and about $0.025 \mathrm{~mm}$ thick. The nanocrystalline state was obtained by a heat treatment in protected atmosphere at $540{ }^{\circ} \mathrm{C}$ for $1 \mathrm{~h}$. 
The magnetic properties were measured by computercontrolled magnetometer using two fluxgate type probes for measuring the stray field of the sample. For purpose of our study we have measured the hysteresis loops at room temperature. The data obtained from descending branch of hysteresis loop were fitted by formulae (1)(3). For fitting procedure, we have used the least square fitting module of Origin software. Subsequently, the hysteresis loop components were constructed from obtained fitting parameters.

\section{Results and discussion}

The experimental data (marked as measured) and the calculated components as they result from our modeling (marked by DWM, DWAN and DR) for amorphous and nanocrystalline sample are presented in Fig. 1 and 2, respectively.

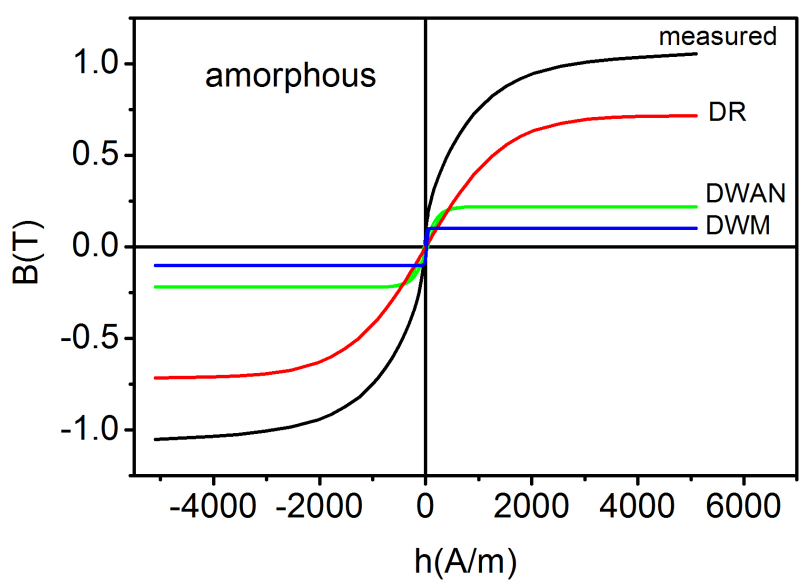

Fig. 1. Measured and calculated (DR, DWAN, DWM) hysteresis loops for amorphous FINEMET.

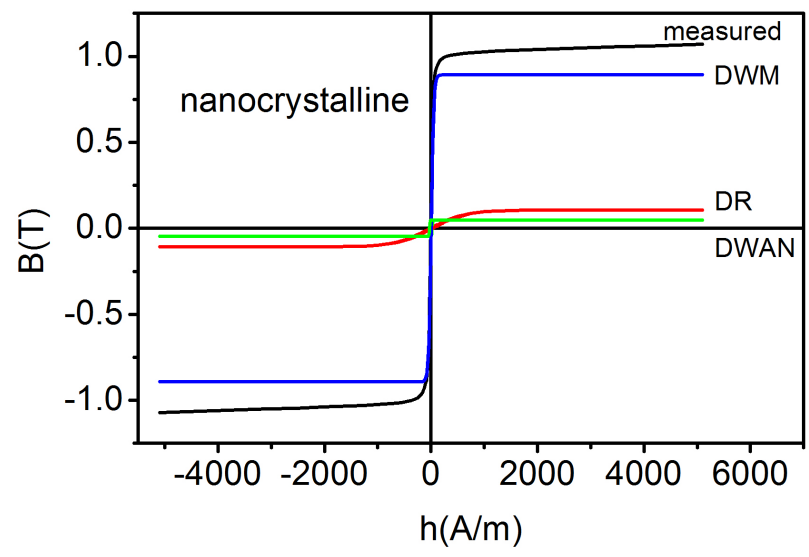

Fig. 2. Measured and fitted (DR, DWAN, DWM) hysteresis loops for nanocrystalline FINEMET.
The component loops obtained by fitting calculations can be assessed to particular magnetization processes: (i) DWM, (ii) DWAN and DR. The peak magnetization of a component can be taken as a measure of the contribution of that particular magnetization process to the overall magnetization. Concerning the amorphous material, perusal of Fig. 1 shows that the DR component dominates over the DWM and DWAN processes which appear in the small field region only. In case of nanocrystalline sample (see Fig. 2) the situation is quite different. The DWM component is the dominant process over the other processes.

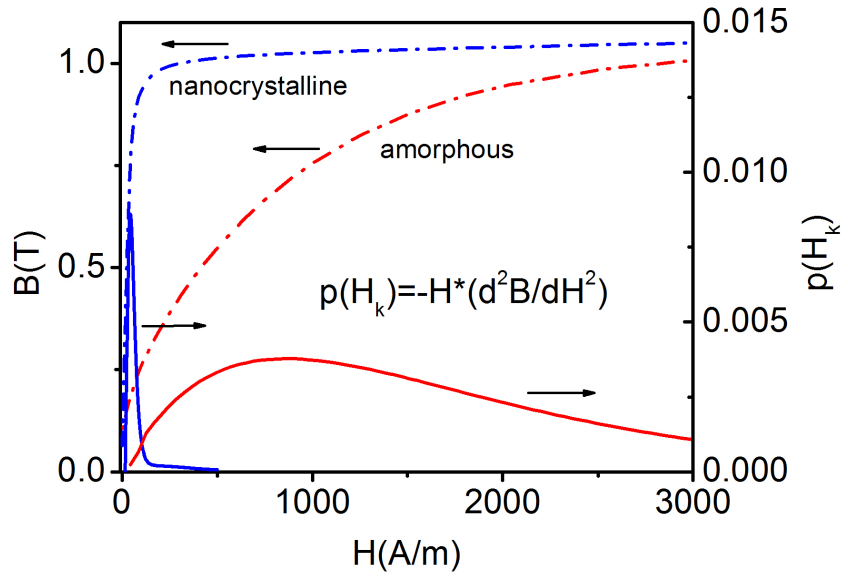

Fig. 3. The distribution of the anisotropy fields for amorphous and nanocrystalline FINEMET (straight lines) calculated for increasing part of the measured hysteresis loops (step lines).

These differences in the magnetization processes of amorphous and nanocrystalline states are connected to the local and average anisotropies of these states. In amorphous state the local magnetostrictive anisotropies originating from the residual thermal stresses $(\sigma)$ induced during the rapid quenching process extend over larger distance than the exchange length and consequently are not averaged out by the exchange interaction. The magnetization process takes place as a rotation of magnetization against these local anisotropy field $\left(m \sim M_{s} / H_{k}\right.$, where anisotropy field $H_{k}=(3 / 2) \lambda \sigma$ and $\lambda$ is the magnetostrictive constant in the amorphous state $(\sim 30 \mathrm{ppm}))$. In contrast to the amorphous state, the nanocrystalline sample present very low magnetic anisotropy [11] (the internal stresses are removed by thermal treatment) and consequently the DWM process is much easier and the corresponding hysteresis loops are narrowed with low coercivity. The differences in magnetization processes can be expressed also in terms of the distribution of the anisotropy fields (see Fig. 3) using the procedure of Barandiaran [12]:

$$
p\left(H_{k}\right)=-H\left(\frac{\mathrm{d}^{2} B}{\mathrm{~d} H^{2}}\right) .
$$

One can see that in the case of amorphous state the 
distribution is wide spread with relatively high value of maximum $H_{k}=860 \mathrm{~A} / \mathrm{m}$ while for nanocrystalline one, the distribution is very sharp with very low value of $H_{k}=43.3 \mathrm{~A} / \mathrm{m}$.

\section{Conclusions}

Our study of the magnetization processes in amorphous and nanocrystalline FINEMET samples showed that hyperbolic model is powerful tool for analysis of these processes in detail. It was clearly demonstrated the role of internal stresses introduced during the process of preparation determining the rotational process to dominate in the amorphous state. In the nanocrystalline state the DWM contribution dominates over the DR and DWAN components. The difference in magnetization processes of the two states are evidenced by the distribution of the anisotropy fields as well: for amorphous state, we have a wide distribution with a mean value of $860 \mathrm{~A} / \mathrm{m}$ whereas for nanocrystalline state we have got a sharp distribution around $43 \mathrm{~A} / \mathrm{m}$.

\section{Acknowledgments}

This work was supported by the projects VEGA of the Scientific Grant Agency of the Ministry for Education of the Slovak Republic No. 1/0164/16, 1/0377/16 and $1 / 0413 / 15$ and by the project "Centre of Excellence for Integrated Research \& Exploitation of Advanced Materials and Technologies in Automotive Electronics" ITMS 26220120055.

\section{References}

[1] F. Preisach, Z. Phys. 94, 277 (1935).

[2] D. Jiles, D.L.C. Atherton, J. Appl. Phys. 55, 2115 (1984).

[3] J. Takacs, Mathematics of Hysteretic Phenomena, Wiley-VCH, Berlin 2003.

[4] D. Jiles, in: Introduction to Magnetism and Magnetic Materials, 2nd ed., Chapman \& Hall, London 1998.

[5] A. Ivanyi, Hysteresis Models in Electromagnetic Computation, Akademiai Kiado, Budapest 1997.

[6] L.K. Varga, Gy. Kovacs, J. Takacs, J. Magn. Magn. Mater. 320, L26 (2008).

[7] J. Takacs, Gy. Kovács, L.K. Varga, J. Magn. Magn. Mater. 320, e1016 (2008).

[8] J. Takacs, COMPEL 20, 1002 (2001).

[9] L.K. Varga, Gy. Kovacs, J. Takacs, J. Magn. Magn. Mater. 320, e814 (2008).

[10] J. Takacs, Gy. Kovacs, L.K. Varga, Physica B 403, 2293 (2008).

[11] G. Herzer, Phys. Scr. T 49, 307 (1993).

[12] J.M. Barandiaran, M. Vazquez, A. Hernando, J. Gonzalez, G. Rivero, IEEE Trans. Magn. 25, 3330 (1989). 